\title{
Joint interpretation of enantiomer and stable isotope fractionation for chiral pesticides
} degradation

\author{
Jin, Biao; Rolle, Massimo
}

Published in:

Water Research

Link to article, DOI:

10.1016/j.watres.2016.08.057

Publication date:

2016

Document Version

Peer reviewed version

Link back to DTU Orbit

Citation (APA):

Jin, B., \& Rolle, M. (2016). Joint interpretation of enantiomer and stable isotope fractionation for chiral pesticides degradation. Water Research, 105, 178-186. https://doi.org/10.1016/j.watres.2016.08.057

\section{General rights}

Copyright and moral rights for the publications made accessible in the public portal are retained by the authors and/or other copyright owners and it is a condition of accessing publications that users recognise and abide by the legal requirements associated with these rights.

- Users may download and print one copy of any publication from the public portal for the purpose of private study or research.

- You may not further distribute the material or use it for any profit-making activity or commercial gain

- You may freely distribute the URL identifying the publication in the public portal

If you believe that this document breaches copyright please contact us providing details, and we will remove access to the work immediately and investigate your claim. 
This is a Post Print of the article published on line $30^{\text {th }}$ August 2016 in Water Research, 105, 178186. The publishers' version is available at the permanent link:

http://dx.doi.org/10.1016/j.watres.2016.08.057

\title{
Joint interpretation of enantiomer and stable isotope fractionation for chiral pesticides degradation
}

\author{
Biao Jin ${ }^{\mathrm{a}}$ and Massimo Rolle $\mathrm{a}^{\mathrm{a}, *}$
}

${ }^{a}$ Department of Environmental Engineering, Technical University of Denmark, Miljøvej Building 113, DK-2800 Kgs. Lyngby, Denmark

* Corresponding author phone: +45 45251566; e-mail: masro@env.dtu.dk

\section{Highlights}

- Integrated model for concentrations, enantiomers and stable isotopes fractionation

- Joint quantitative approach to interpret dual enantiomer and stable isotope data

- Characterization of isotope and enantiomer selective reaction mechanisms

- Model validation based on chiral pesticides degradation data 


\section{Abstract}

2 Chiral pesticides are important contaminants affecting the health and functioning of aquatic systems.

3 The combination of stable isotope and enantiomer analysis techniques has been recently proposed

4 to better characterize the fate of these contaminants in natural and engineered settings. We

5 introduce a modeling approach with the aim of unifying and integrating the interpretation of

6 isotopic and enantiomeric fractionation. The model is based on the definition of enantiomer-specific

7 isotopologues and jointly predicts the evolution of concentration, enantiomer fractionation, as well

8 as changes in stable isotope ratios of different elements. The method allows evaluating different

9 transformation pathways and was applied to investigate enzymatic degradation of dichlorprop

10 (DCPP), enzymatic degradation of mecoprop methyl ester (MCPPM), and microbial degradation of

$11 \alpha$-hexachlorocyclohexane $(\alpha-\mathrm{HCH})$ by different bacterial strains and under different redox

12 conditions. The model accurately reproduces the isotopic and enantiomeric data observed in

13 previous experimental studies and precisely captures the dual-dimensional trends characterizing

14 different reaction pathways. Furthermore, the model allows testing possible combinations of

15 enantiomer analysis (EA), compound specific isotope analysis (CSIA), and enantiomer specific 16 isotope analysis (ESIA) to identify and assess isotope and enantiomer selective reaction 17 mechanisms. 


\section{Introduction}

Organic pesticides are applied in many anthropogenic activities and constitute an increasing threat to the quality and health of aquatic systems (Fenner et al., 2013; Schwarzenbach et al., 2006). Pesticides are frequently detected in drinking water wells (Spliid and Køppen, 1998; Turner et al., 2006; Vorkamp et al., 2014) and are a primary reason causing the shutdown of drinking water supplies (e.g., Thorling, 2015). Pesticides can bypass wastewater treatment plants and enter natural aquatic systems (Lapworth et al., 2012; McKnight et al., 2015; Pal et al., 2010) posing serious risks to aquatic life and human health (Schwarzenbach et al., 2010). Chiral compounds represent an important fraction of organic pesticides released in the environment (Wong, 2006; Zipper et al., 1998). These chemicals are dispensed as mixtures of two enantiomers (i.e., a pair of molecular entities which are mirror images of each other and nonsuperposable, IUPAC 2014). They are of special interest and concern due to the fact that stereoisomers of one chiral compound often have different biological fate and toxic effects (Bollmann et al., 2014; Petrie et al., 2014). Thus, detailed understanding of the environmental distribution and the degradation processes of chiral pesticides is essential for risk assessment and for evaluating the hazardous effects of these organic compounds in both wastewater treatment systems and natural aquatic environments (Stenzel et al., 2013; Wong, 2006).

Due to the complexity in assessing the fate of organic pollutants in environmental systems, where intricate and coupled physical and biochemical processes hinder quantitative evaluations, it is necessary to combine conventional and innovative approaches. Concentration analyses of mother compounds and their metabolites are typically applied and represent a primary source of information. However, this approach is often not conclusive, since it is hampered by the difficulty to distinguish between transformation and dilution processes. For chiral compounds, enantiomer 
analysis (EA) is an additional valuable tool to demonstrate the occurrence of biotransformation processes (Rügge et al., 2002). This approach analyzes changes of enantiomeric compositions occurring during enantioselective biochemical transformations. Another independent approach is compound specific isotope analysis (CSIA) which determines the isotopic evolution during degradation processes. Recently, stable isotope techniques have been developed at a fast pace and applied in various experimental (e.g., Sakaguchi-Söder et al., 2007, Bashir et al., 2015; Elsayed et al., 2014; Jin et al., 2014; Rolle et al., 2010; Schmidt and Jochmann, 2012) and modeling studies (e.g., Prommer et al., 2009; Eckert et al., 2012; Jin et al., 2013; Thullner et al., 2012; Van Breukelen and Rolle, 2012). A further advance is enantiomer specific isotope analysis (ESIA), which allows analyzing the isotopic composition of individual enantiomers (Badea et al., 2011; Maier et al., 2013). Facilitated by the developments of analytical techniques, a number of recent experimental studies have proposed to combine enantiomer and isotope analyses to investigate the fate of different chiral organic pollutants, including phenoxy acids (Milosevic et al., 2013; Qiu et al., 2014), hexachlorocyclohexane isomers (Badea et al., 2011; Bashir et al., 2013) and phenoxyalkanoic methyl herbicides (Jammer et al., 2014). Such approach has shown great potential to decipher degradation mechanisms of different chiral compounds. In fact, different reaction pathways are characterized and effectively visualized by plotting enantiomer ratios together with stable isotope ratios (Bashir et al., 2013; Jammer et al., 2014; Milosevic et al., 2013; Qiu et al., 2014). During the reaction of chiral organic contaminants, stable isotope fractionation occurs due to mass differences of isotopologues of individual enantiomers. Specifically, the mass differences of isotopologues result in different bond strength, and thus underdo reaction processes at different rates. Enantiomers, instead, have the same mass and bond energy, therefore enantiomer enrichment in a biochemical reaction is due to different geometrical recognitions of the two enantiomer molecules (Jammer et al., 2014; Wong, 2006). Although the fractionation of stable isotope and enantiomers is due to different 
mechanisms, they are intimately related and occur simultaneously. Even though isotope and enantiomer fractionation occur simultaneously during chiral pesticide degradation, the two fractionating systems are evaluated independently and, only subsequently, they are merged in twodimensional representations for mechanistic interpretation. Therefore, a first-principle based modeling approach aiming at unifying the information gained from enantiomer and stable isotope analyses is required. Such development will contribute to improve our capability to interpret combined enantiomer and isotope signals, as well as to overcome some of the challenges emerging from experimental studies in which nonlinear patterns are commonly observed due to the significantly different extents of enantiomer and stable isotope fractionation.

The objective of this work is to provide an integrated evaluation scheme to describe and interpret the evolution of enantiomer and stable isotope ratios during the degradation of chiral pollutants. The proposed framework allows the joint and simultaneous description of: (i) concentrations of parent compounds and metabolites, (ii) enantiomer fractionation, and (iii) stable isotope evolution. The approach is validated with recently published concentration, enantiomer and stable isotope data of important chiral pesticides such as dichlorprop (DCPP), mecoprop methyl ester (MCPPM) and $\alpha$ hexachlorocyclohexane $(\alpha-\mathrm{HCH})$. The model is also used to test the applicability and the potential of different two-dimensional approaches combining stable isotope fractionation (as individual enantiomers or as compound average) with enantiomer analysis to characterize different reaction mechanisms of chiral pesticides degradation.

\section{Material and methods}

\subsection{Enantiomer-specific isotope modeling}

The modeling framework originates from the main idea of incorporating mechanistic information on contaminants degradation in model-based interpretation of stable isotope data (Jin and Rolle, 
2014). A major challenge addressed by the proposed approach is to consistently integrate the quantitative description of enantiomer and stable isotope evolution. Enantiomers are normally denoted according to their molecular configuration $(R$ and $S$ ) or optical activity (+ and -) (Wong, 2006). To illustrate our approach, we consider an example using $R$ and $S$ enantiomers undergoing different extents of degradation and resulting in the enrichment of one enantiomer. An example using the optical activity notation is outlined in the last section of the Supplementary Material. Besides enantioselectivity, the cleavage of chemical bonds during degradation of a chiral compound also causes stable isotope fractionation in both $R$ and $S$ enantiomeric molecules. As many organic micropollutants, chiral organic pesticides often have large molecular size and thus it is efficient to track directly the atoms experiencing isotope effects during transformation processes (Jin and Rolle, 2015). Therefore, it is convenient to define enantiomer-specific isotopologues: enantiomer molecules with different isotopic compositions at reactive positions. The proposed model can be formulated for single- and multi-element isotope fractionation. In the following we illustrate the procedure for a dual-element system. Considering the occurrence of isotopically-sensitive atoms of two elements, $\mathrm{X}$ and $\mathrm{Y}$, the relative abundances of the $j^{\text {th }}$ enantiomer-specific isotopologues for $R$ and $S$ enantiomers are given by the product of the abundance of each isotope (Hofstetter et al., 2007; Sakaguchi-Söder et al., 2007):

$$
\begin{aligned}
& A_{j}^{R}=\prod_{i=1}^{m_{X}}\left(X_{H, i}^{v_{i}} \cdot X_{L, i}^{1-v_{i}}\right) \cdot \prod_{h=1}^{m_{Y}}\left(Y_{H, h}^{u_{h}} \cdot Y_{L, h}^{1-u_{h}}\right) \\
& A_{j}^{S}=\prod_{i=1}^{m_{X}}\left(X_{H, i}^{v_{i}} \cdot X_{L, i}^{1-v_{i}}\right) \cdot \prod_{h=1}^{m_{Y}}\left(Y_{H, h}^{u_{h}} \cdot Y_{L, h}^{1-u_{h}}\right)
\end{aligned}
$$

113 where $A^{S}{ }_{j}$ and $A^{R}{ }_{j}$ are the relative abundances of the $j^{\text {th }}$ enantiomer-specific isotopologue of $R$ and $S$ enantiomer. $A_{j}^{S}$ and $A^{R}{ }_{j}$ are expressed considering exclusively the isotope abundances of atoms $\mathrm{X}$ and $\mathrm{Y}$ at fractionating positions. Each enantiomer isotopologue can contain up to $m_{X}$, total $\mathrm{X}$ atoms, 
and $m_{Y}$, total $\mathrm{Y}$ atoms, at fractionating positions. The indexes $i$ and $h$ identify $\mathrm{X}$ and $\mathrm{Y}$ atoms, 2005):

$$
\begin{aligned}
& \alpha^{R}=A K I E_{R}^{-1} \approx 1+\frac{n_{R}}{m_{R}} \cdot \varepsilon_{\text {bulk }}^{R} \\
& \alpha^{S}=A K I E_{S}^{-1} \approx 1+\frac{n_{S}}{m_{S}} \cdot \varepsilon_{\text {bulk }}^{S}
\end{aligned}
$$

These equations can be written both for $\mathrm{X}$ and $\mathrm{Y}$ isotopes. $\alpha$ is the fractionation factor for the $R$ or $S$ enantiomer, $\varepsilon$ is the bulk enrichment factor of individual enantiomers, $n$ is the total number of atoms of $\mathrm{X}$ or $\mathrm{Y}$ element in one enantiomer molecule, $m$ is the number of atoms of one element located at fractionating positions in the $R$ or $S$ enantiomer. Accurate estimates of AKIE values are important for the proposed modeling approach. Besides the calculations based on Eqs. 3 and 4, the advances in analytical techniques, as well as in theoretical calculations and computational chemistry, are likely to provide more direct insight on AKIEs based on position-specific isotope measurements, as well as on computational chemistry predictions (e.g., Breider and Hunkeler, 2011; Grzybkowska et al., 2014; Świderek and Paneth, 2012; Wuerfel et al., 2013).

We track the concentration evolution of each enantiomer-specific isotopologue according to a specified kinetic rate law. To demonstrate the approach we consider a first-order kinetic, however 
the model is quite general and similar governing equations can be formulated for more complex degradation kinetics, including Michaelis-Menten kinetic coupling the contaminant degradation to biomass dynamics (see details in the Supplementary Material). For a first-order reaction rate, the $j^{\text {th }}$ enantiomer-specific isotopologues of $R$ and $S$ enantiomers can be defined as:

$$
\begin{aligned}
& r_{j}^{R}=k_{R} \cdot C_{j}^{R} \cdot \prod_{i=1}^{m_{X}}\left(\alpha_{X, i}^{R}\right)^{v_{i}} \cdot \prod_{h=1}^{m_{Y}}\left(\alpha_{Y, h}^{R}\right)^{u_{h}} \\
& r_{j}^{S}=k_{S} \cdot C_{j}^{S} \cdot \prod_{i=1}^{m_{X}}\left(\alpha_{X, i}^{S}\right)^{v_{i}} \cdot \prod_{h=1}^{m_{Y}}\left(\alpha_{Y, h}^{S}\right)^{u_{h}}
\end{aligned}
$$

where $r_{j}$ is the reaction rate for the $j^{\text {th }}$ enantiomer-specific isotopologue of $R$ or $S$ enantiomer, $\alpha$ is the fractionation factor as defined in Eqs. 3 and $4, C_{j}$ is the concentration of the $j^{\text {th }}$ enantiomerspecific isotopologue, and $m, n, v$ and $u$ are defined in Eqs. 1 and 2.

The concentration change of $R$ and $S$ enantiomers is described by tracking each enantiomer-specific isotopologue, respectively:

$$
\begin{aligned}
& \frac{d C_{j}^{R}}{d t}=-r_{j}^{R} \\
& \frac{d C_{j}^{S}}{d t}=-r_{j}^{S}
\end{aligned}
$$

where $C^{R}{ }_{j}$ and $C^{S}{ }_{j}$ are the concentrations of the $j^{\text {th }} R$ and $S$ enantiomer-specific isotopologues, $t$ is the time, and $r_{j}$ is the reaction rate of the $j^{\text {th }}$ enantiomer-specific isotopologue. The concentrations of individual enantiomers are obtained by summing the concentration of each enantiomer-specific isotopologue. The initial concentrations of the enantiomer-specific isotopologues are the product of the initial total concentration of $R$ and $S$ enantiomers with the corresponding initial abundances (Eqs. 1 and 2). 
152 The enantiomer ratio $(E R)$ and the enantiomer fraction $(E F)$ are normally used to describe 153 enantiomer enrichment of chiral compounds (Harner et al., 2000; Jammer et al., 2014; Qiu et al., 154 2014). In the proposed framework, $E R$ and $E F$ can be computed considering the concentrations of 155 enantiomer-specific isotopologues:

$$
\begin{gathered}
E R=\frac{C_{S}}{C_{R}}=\frac{\sum_{j=1}^{N} C_{j}^{S}}{\sum_{j=1}^{N} C_{j}^{R}} \\
E F_{R}=\frac{C_{R}}{C_{R}+C_{S}}=\frac{\sum_{j=1}^{N} C_{j}^{R}}{\sum_{j=1}^{N} C_{j}^{R}+\sum_{j=1}^{N} C_{j}^{S}} \\
E F_{S}=\frac{C_{S}}{C_{R}+C_{S}}=\frac{\sum_{j=1}^{N} C_{j}^{S}}{\sum_{j=1}^{N} C_{j}^{R}+\sum_{j=1}^{N} C_{j}^{S}}
\end{gathered}
$$

156

where $C_{R}$ and $C_{S}$ are the concentrations of individual enantiomers, $C_{j}$ is the concentration of each enantiomer-specific isotopologue for $\mathrm{R}$ or $\mathrm{S}$ enantiomer, and $N$ is the total number of enantiomer-

159 specific isotopologues.

160 The isotope ratios of elements $\mathrm{X}$ and $\mathrm{Y}$ at positions experiencing isotope effects in $R$ or $S$

161 enantiomer-specific isotopologues are calculated by considering the total number of heavy and light isotopes (Jin et al., 2011), and are expressed as:

$$
R_{X, R}^{\prime}=\frac{\operatorname{Tot}\left(\mathrm{X}_{\mathrm{H}}\right)}{\operatorname{Tot}\left(\mathrm{X}_{\mathrm{L}}\right)}=\frac{C_{j}^{R} \cdot \sum_{i=1}^{m_{X}} v_{i}}{C_{j}^{R} \cdot \sum_{i=1}^{m_{X}}\left(1-v_{i}\right)}
$$




$$
\begin{gathered}
R_{X, S}^{\prime}=\frac{\operatorname{Tot}\left(\mathrm{X}_{\mathrm{H}}\right)}{\operatorname{Tot}\left(\mathrm{X}_{\mathrm{L}}\right)}=\frac{C_{j}^{S} \cdot \sum_{i=1}^{m_{X}} v_{i}}{C_{j}^{S} \cdot \sum_{i=1}^{m_{X}}\left(1-v_{i}\right)} \\
R_{Y, R}^{\prime}=\frac{\operatorname{Tot}\left(\mathrm{Y}_{\mathrm{H}}\right)}{\operatorname{Tot}\left(\mathrm{Y}_{\mathrm{L}}\right)}=\frac{C_{j}^{R} \cdot \sum_{h=1}^{m_{Y}} u_{h}}{C_{j}^{R} \cdot \sum_{h=1}^{m_{Y}}\left(1-u_{h}\right)} \\
R_{Y, S}^{\prime}=\frac{\operatorname{Tot}\left(\mathrm{Y}_{\mathrm{H}}\right)}{\operatorname{Tot}\left(\mathrm{Y}_{\mathrm{L}}\right)}=\frac{C_{j}^{S} \cdot \sum_{h=1}^{m_{Y}} u_{h}}{C_{j}^{S} \cdot \sum_{h=1}^{m_{Y}}\left(1-u_{h}\right)}
\end{gathered}
$$

163 in which $R_{X}^{\prime}$ and $R_{Y}^{\prime}$ are the isotope ratios of all the $\mathrm{X}$ and $\mathrm{Y}$ atoms on isotopically-sensitive 164 positions of each enantiomer at a given point in time, $C_{j}$ is the concentration of the $j^{\text {th }}$ enantiomer165 specific isotopologue at that point in time, and $m, v$ and $u$ are defined in Eqs. 1 and 2.

166 Enantiomer-specific isotope ratios (i.e., stable isotope ratios of individual enantiomers) are 167 calculated at each point in time by taking into account changes of stable isotope ratios at 168 fractionating positions and the dilution effects from non-fractionating positions in the enantiomer 169 molecules as well as the initial bulk isotope ratio $R_{0}$ :

$$
\begin{aligned}
& R_{X, R}=R_{X, R}^{\prime} \cdot \frac{m_{X}}{n_{X}}+R_{0, X} \cdot \frac{n_{X}-m_{X}}{n_{X}} \\
& R_{X, S}=R_{X, S}^{\prime} \cdot \frac{m_{X}}{n_{X}}+R_{0, X} \cdot \frac{n_{X}-m_{X}}{n_{X}} \\
& R_{Y, R}=R_{Y, R}^{\prime} \cdot \frac{m_{Y}}{n_{Y}}+R_{0, Y} \cdot \frac{n_{Y}-m_{Y}}{n_{Y}}
\end{aligned}
$$




$$
R_{Y, S}=R_{Y, S}^{\prime} \cdot \frac{m_{Y}}{n_{Y}}+R_{0, Y} \cdot \frac{n_{Y}-m_{Y}}{n_{Y}}
$$

171 where $R$ is the enantiomer-specific isotope ratio of element $\mathrm{X}$ or $\mathrm{Y}, R$ ' is the corresponding isotope ratios of atoms at fractionating positions as computed in Eqs. 12-15.

173 Although a few recent contributions reported enantiomer-specific isotope ratios (ESIA), bulk isotope ratios determined by compound specific isotope analysis (CSIA) are commonly measured in most experimental studies. Bulk isotope ratios can be related to enantiomer-specific isotope ratios by the following weighted averages:

$$
\begin{gathered}
R_{X}=R_{X, R} \cdot E F_{R}+R_{X, S} \cdot E F_{S} \\
R_{Y}=R_{Y, R} \cdot E F_{R}+R_{Y, S} \cdot E F_{S}
\end{gathered}
$$

where $R_{X}$ and $R_{Y}$ are the bulk isotope ratios of a chiral organic compound, $R_{X, R}, R_{X, S}, R_{Y, R}$ and $R_{Y, S}$ are the enantiomer-specific isotope ratios and $E F_{R}$ and $E F_{S}$ are the enantiomer fractions of individual enantiomers as defined in Eqs. 10 and 11.

The proposed model is developed in MATLAB ${ }^{\circledR}$ and it is applied to describe contaminant degradation in batch systems. The governing equations presented above (Eqs. 5-8) are solved for three selected illustrative examples of chiral compounds degradation. The specific derivation of the governing equations for the first illustrative example is described in the Supplementary Material. The document also provides a table summarizing the enantiomer-specific isotopologues and their number for the different examples considered. In all cases the reaction kinetics, described with a first-order or with a Michaelis-Menten formulation, were fitted to the concentration data provided in recently published experimental studies. The trust-region-reflective method implemented in the MATLAB ${ }^{\circledR}$ function lsqnonlin was used to minimize the sum of normalized squared errors between the observed and simulated concentration data. Enantiomer ratios and fractions, as well as 
stable isotope ratios, were not fitted but evaluated with Eqs. 9-21 and directly compared to the experimental results. Details on the fitting procedure, as well as an overview of the fitted parameters are also available in the Supplementary Material.

\subsection{Examples of chiral pesticides degradation}

We test our modeling approach considering the degradation of three important chiral pesticides. These examples are enzymatic degradation of dichlorprop (DCPP), enzymatic degradation of mecoprop methyl ester (MCPPM) and aerobic and anaerobic biodegradation of $\alpha$ hexachlorocyclohexane $(\alpha-\mathrm{HCH})$. The chemical structures, the reaction mechanisms, the target stable isotopes and the reactive bonds of these chiral compounds are illustrated in Table 1. Recent experimental studies have provided high-quality data on enantioselectivity as well as on compound specific (CSIA) and enantiomer specific stable isotope analysis (ESIA) for these chemicals and are used to validate the joint quantitative approach proposed in this study.

Dichlorprop (DCPP) is a phenoxy acid commonly used as herbicide to control weeds. It is frequently detected in groundwater systems (Spliid and Køppen, 1998). Enantiomer fractionation and enantiomer specific carbon isotope fractionation have been observed during enzymatic degradation of DCPP by enzyme RdpA from Sphingobium herbicidovorans MH (Qiu et al., 2014). In order to investigate enantiomer-specific degradation mechanisms of DCPP, a two-dimensional approach combining enantiomer analysis (EA) and enantiomer specific isotope analysis (ESIA) has been applied. The data provided for DCPP degradation were used to validate the capability of the proposed modeling approach to simultaneously predict the evolution of R- and S-DCPP enantiomer concentrations, the formation of the reaction product, as well as the joint evaluation of C-ESIA isotope ratios and enantiomer fractionation. 
213 Mecoprop methyl ester (MCPPM) is a phenoxyalkanoic methyl herbicide, which is a contaminant 214 frequently found in aquatic environments. Enantiomer fractionation and compound specific isotope 215 fractionation (CSIA) of this chemical have been recently observed during enzymatic reactions by 216 different types of lipase enzymes from distinct microbial strains including Pseudomonas 217 fluorescens, Candida rugose and Pseudomonas cepacia (Jammer et al., 2014). This dataset was of 218 interest since it allowed testing the ability of the integrated model to characterize distinct reaction 219 pathways by combining enantiomer analysis (EA) and bulk (i.e., compound average) isotope ratios 220 from compound specific isotope analysis (CSIA).

221 The third and final application is focused on biodegradation of $\alpha-H e x a c h l o r o c y c l o h e x a n e ~(\alpha-H C H)$. $\alpha-\mathrm{HCH}$ is one of the dominant byproducts during the production of Lindane $(\gamma-\mathrm{HCH})$, a widely produced and applied insecticide (Lal et al., 2010; Phillips et al., 2005). Carbon ESIA and enantiomer analysis (EA) have been applied to investigate biodegradation of $\alpha$-HCH by different microbial strains including S. indicum strain B90A, S. japonicum strain UT26 and Clostridium pasterianum (Badea et al., 2011; Bashir et al., 2013). Biodegradation of $\alpha-\mathrm{HCH}$ occurs under both aerobic and anaerobic conditions, involving dehydrochlorination and dichloro-elimination, respectively. We applied our model to reproduce the observed enantiomer and stable isotope signals 229 and to differentiate enantiomer-specific degradation pathways of $\alpha$-HCH. Besides the 230 experimentally investigated combination of C-ESIA with EA (Bashir et al., 2013), the validated model has been used to explore the potential of another combined two-dimensional approach (i.e., C-CSIA combined with EA) to characterize different isotope and enantiomer selective reaction mechanisms.

Table 1. Chemical structures, enantiomers, stable isotopes and reaction mechanisms for the considered chiral 236 organic pesticides. 


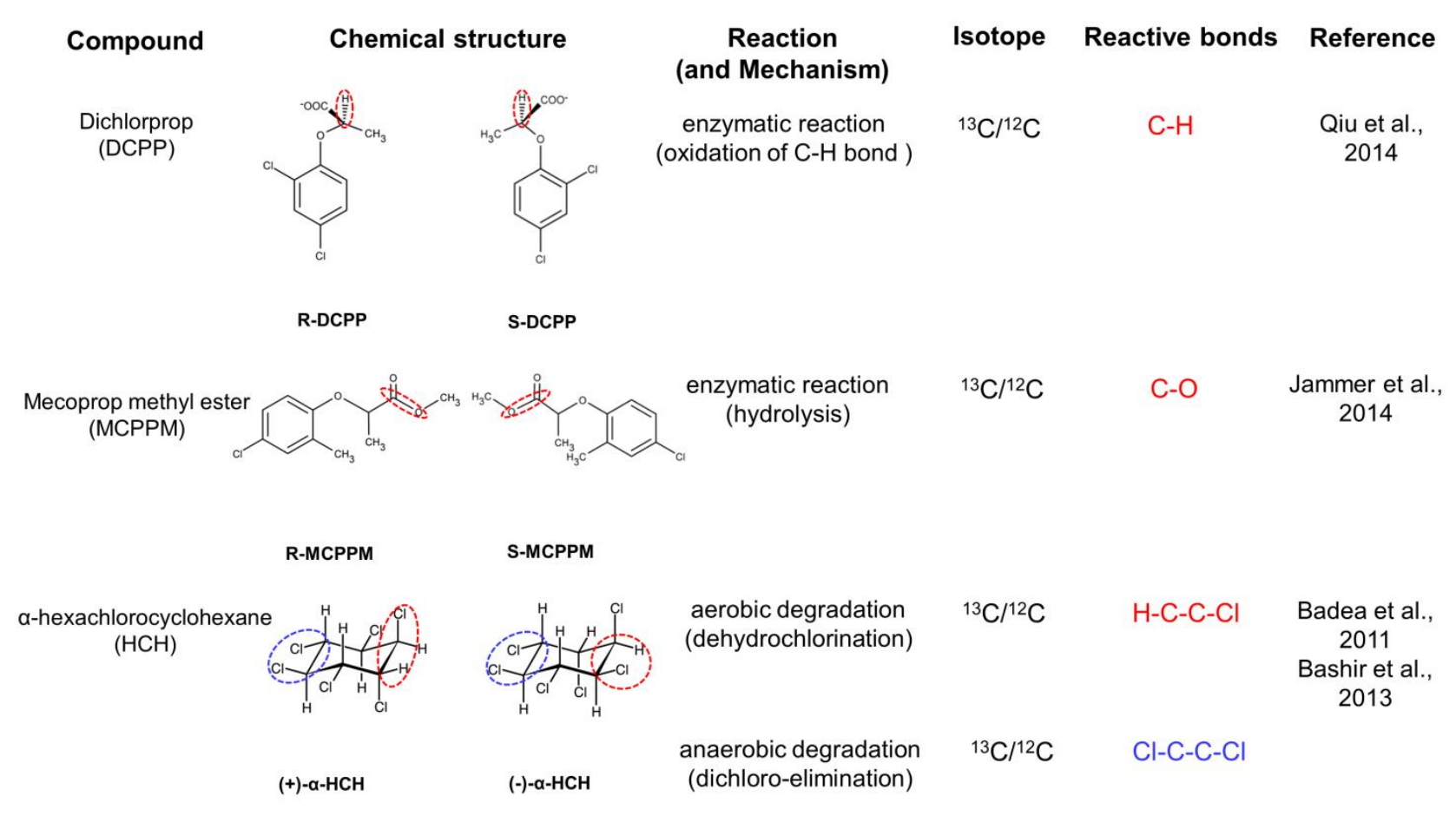

\section{Results and discussion}

\subsection{Enzymatic degradation of dichlorprop (DCPP)}

Experimental data on concentration evolution of DCPP and its main metabolite (phenol), enantioselective effects and enantiomer specific carbon isotope fractionation were measured during degradation of this pesticide by enzyme RdpA from Sphingobium herbicidovorans MH (Qiu et al., 2014). The reported experimental observations are shown as symbols in Fig. 1 together with the outcomes of the simulations using the proposed integrated approach (solid lines). The enantiomer R-DCPP (blue) is consumed according to a first-order degradation kinetic with a reaction rate constant $k_{R}=0.038 \pm 0.003 \mathrm{~min}^{-1}$, forming phenol (gray) as metabolite. The concentration of the other 250 isomer (S-DCPP), instead, remains constant due to the selectivity of the RdpA enzymes that 251 exclusively degrade the $\mathrm{R}$ enantiomer (Fig. 1a). The model accurately captures both the 
consumption of the chiral pesticide degradation and the production of the metabolite. Also the 253 isotopic signals observed during enzymatic degradation of DCPP are considerably different 254 between $\mathrm{R}$ and $\mathrm{S}$ enantiomers. In fact, significant carbon isotope fractionation occurs only for R255 DCPP, varying from $-25.1 \%$ to $-22.5 \%$, whereas no significant carbon isotope fractionation 256 occurs for S-DCPP. Fig. 1b illustrates the observed and simulated temporal trends of carbon isotope 257 ratios for both DCPP enantiomers. The model reproduces satisfactorily the linear increase of $\delta^{13} \mathrm{C}$ 258 observed for R-DCPP as well as the stable isotopic composition of S-DCPP. Illustrative plots are 259 also obtained by representing the stable carbon isotope signature as a function of the enantiomer 260 fraction (Fig. 1c). The fast consumption of R-DCPP leads to decreasing R-enantiomer fraction 261 (from 50\% to 4\%) and increasing S-enantiomer fraction (50\% to 96\%). Interestingly, a nonlinear 262 relationship is observed for R-DCPP due to the considerably larger extent of enantiomer 263 fractionation compared to the relatively small carbon isotope fractionation. This behavior is 264 accurately predicted by the model, which results in a concave upward profile with increasing 265 steepness as the reaction proceeds and the fraction of R-DCPP progressively decreases. The 266 quantitative interpretation of the experimental data with the proposed integrated modeling approach 267 allows simultaneously and accurately capturing the concentrations, stable isotopes and 268 enantioselective behavior observed in the experimental study. A specific advantage that is worth 269 noticing is the good performance of the model when its outcomes are compared to the experimental 270 data in the two-dimensional plot combining stable isotopes and enantiomer fractionation. 

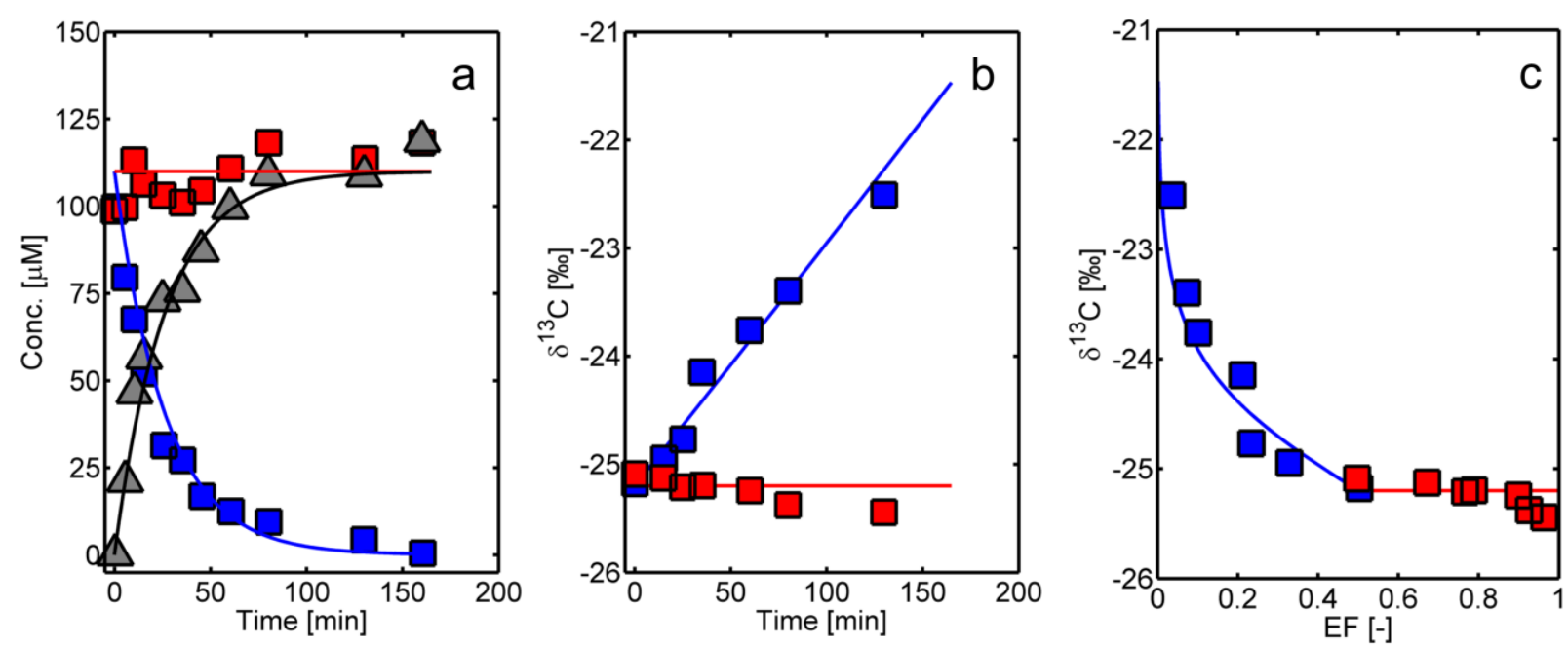

R-DCPP measured $\square$ S-DCPP measured $\triangle$ phenol measured — R-DCPP simulated — S-DCPP simulated $\longrightarrow$ phenol simulated

Figure 1. Isotope and enantiomer fractionation during enzymatic degradation of dichlorprop (DCPP). The symbols represent experimental data reported by Qiu et al., 2014 and the solid lines are simulation results: (a) concentration change of R- (blue squares) and S- (red squares) enantiomers of DCPP; (b) carbon isotope fractionation for both R- and S-DCPP; and (c) two-dimensional plot combining carbon isotope and enantiomer fractionation.

\subsection{Enzymatic hydrolysis of mecoprop methyl ester (MCPPM)}

Enantiomer and carbon isotope fractionation have been observed during enzymatic hydrolysis of a phenoxyalkanoic methyl herbicide, mecoprop methyl ester (MCPPM) (Jammer et al., 2014). The enzymatic reactions by different types of lipase enzymes, Pseudomonas fluorescens, Candida rugose and Pseudomonas cepacia were investigated combining compound specific stable isotope analysis (C-CSIA) with enantiomer analysis (EA). We simulate MCPPM degradation with a firstorder kinetic model according to the unified framework outlined in Section 2 to integrate the quantitative description of enantioselectivity and stable isotope fractionation. The governing equations for the $\mathrm{S}$ and $\mathrm{R}$ enantiomers of MCPPM and the concentration evolution in the experiments conducted with the enzymes of the three different strains are reported in the Supplementary Material. Two-dimensional plots combining stable isotope and enantiomer fractionation are shown in Fig. 2. Notice that enantiomer fractionation is expressed as enantiomer 
ratio, $E R$, since CSIA data do not allow discriminating between the different enantiomers and, thus, can only be presented as a function of $E R$ rather than of the enantiomer fraction, $E F$. The carbon isotope fractionation of MCPPM occurs at similar extents for the three enzymatic reactions, where the following shifts in $\delta^{13} \mathrm{C}$ values were observed: $4.7 \%$ for Pseudomonas cepacia, $5.2 \%$ for Pseudomonas fluorescens and $5.6 \%$ for Candida rugose. Therefore, in this case, C-CSIA alone is not conclusive to clearly identify the three different enzymatic reactions. To this end, a twodimensional approach combining C-CSIA with enantiomer analysis is highly beneficial and was proposed in the experimental study (Jammer et al., 2014). In fact, distinct enantiomer enrichments are observed for the three investigated reactions. Combining enantiomer fractionation and C-CSIA signals allows distinguishing and clearly visualizing the three enzymatic reactions (Fig. 2a). Similarly to what has been observed for DCPP degradation, nonlinear relationships between compound specific carbon isotope ratios and enantiomeric ratios are also observed during enzymatic reactions of MCPPM. This is due to the much more significant changes of enantiomer ratios that are three orders of magnitude larger than the shifts in stable isotope ratios. The nonlinear behavior is well captured by the outcomes of the model that accurately reproduce the different trends observed for the three enzymatic reactions. The model results show bending trends that are more pronounced for the enzymatic reactions by Candida rugose and Pseudomonas fluorescens, which are characterized by more extensive enantiomer fractionation. The profiles characterizing the reaction mechanisms progressively become less steep at later reaction times and show extents of slope variations of $85 \%$ for Pseudomonas cepacia, 93\% for Pseudomonas fluorescens and for 98\% for Candida rugose. An additional direct outcome of the proposed modeling approach is the quantification of isotope ratios directly at the position experiencing isotope effects. This naturally stems from the model formulation based on enantiomer-specific isotopologues and its capability to track isotope ratios at isotopically sensitive positions (Eqs. 12-15). Position-specific isotope data 
were not available for MCPPM degradation, but recent advances have shown the capability of measuring changes of isotope ratios at specific molecular positions of certain organic compounds (e.g., Wuerfel et al., 2013) and we expect that a number of future investigations will provide such data for a wide range of organic pollutants. In Fig. $2 \mathrm{~b}$ we present modeling results to describe position-specific isotope fractionation occurring at the reactive carbon atom during enzymatic hydrolysis of MCPPM combined with the corresponding enantiomer ratios. The three distinct reactions are clearly identified in the two-dimensional plot with the advantage that the carbon isotope fractionation reported in the ordinate axis is now characterized by a larger magnitude (i.e., $60 \%$ for Pseudomonas cepacia, $66 \%$ for Pseudomonas fluorescens and $75 \%$ for Candida rugose), since the model directly predicts the fractionation at reactive position without the dilution effects of other non-reactive carbon atoms present in the pesticide molecule.
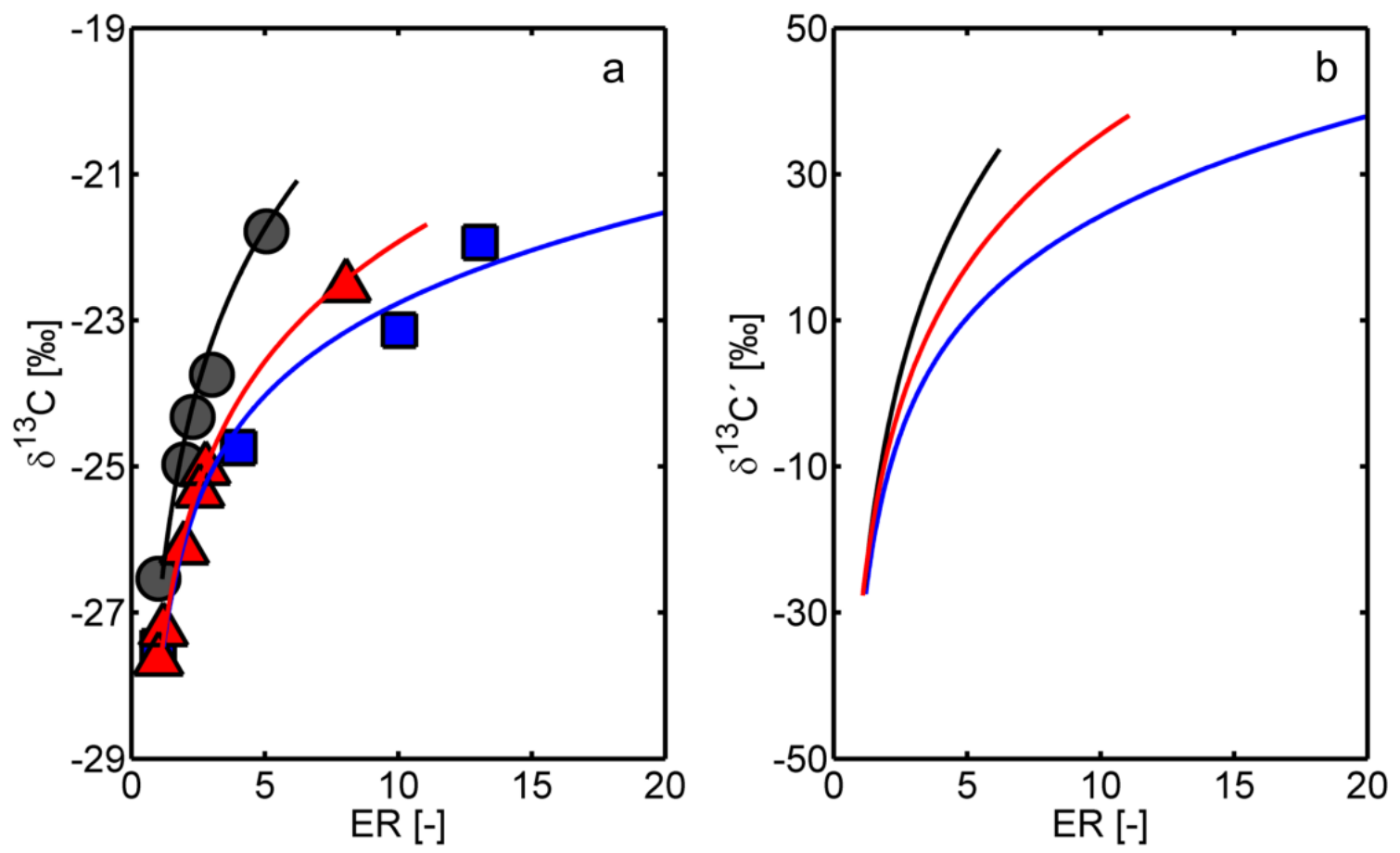

Figure 2. Isotope and enantiomer fractionation during enzymatic degradation of mecoprop methyl ester enantiomer ratios; (b) simulated position-specific isotope fractionation as function of the enantiomer ratio. 


\subsection{Aerobic and anaerobic biodegradation of $\alpha$-hexachlorocyclohexane ( $\alpha-\mathrm{HCH})$}

332 As last illustrative example to validate the proposed approach, we consider biodegradation of 333 hexachlorocyclohexane which has been experimentally studied combining enantiomer analysis and enantiomer-specific isotope analysis, ESIA (Badea et al., 2011; Bashir et al., 2013). The degradation of $\alpha-\mathrm{HCH}$ by different microbial strains including S. indicum strain B90A, $S$. japonicum strain UT26 and Clostridium pasterianum was investigated in batch culture experiments.

We provide a quantitative, model-based interpretation of the experimental data reported in the study of Bashir et al. 2013. We describe the observed concentration trends during $\alpha$-HCH biodegradation with Michaelis-Menten kinetics coupled to the dynamics of growth and decay of the different microbial strains (Supplementary Material). The modeling framework outlined above was adopted to jointly describe the evolution of the two $\alpha-\mathrm{HCH}$ enantiomers (identified by their optical activity: + and - ) and the enantiomer-specific carbon isotope fractionation. As shown in Fig. 3a, the reactions by different microbial strains are clearly identified in the two-dimensional plot. Aerobic degradation by S. indicum strain B90A (squares) and S. japonicum strain UT26 (triangles) as well as the anaerobic biodegradation by Clostridium pasterianum (circles) yield different extents of both enantiomer and enantiomer specific carbon isotope fractionation. Anaerobic biodegradation of $\alpha$ $\mathrm{HCH}$ resulted in significant carbon isotope fractionation (by $6.0 \%$ and $3.2 \%$ for $(+)$ and (-) $\alpha-$ $\mathrm{HCH}$, respectively), but almost no enantiomer fractionation was observed comparing with the aerobic reactions. This indicates that the enzymes involved in the anaerobic degradation of $\alpha-\mathrm{HCH}$ are rather isotopically-sensitive than enantiomer selective. Although the aerobic biodegradation by the two different strains have the same reaction mechanisms involving dehydrochlorination (Table 1), different extents of enantiomer fractionation were observed. The enantiomer fraction of $\alpha-\mathrm{HCH}$ varies by $17 \%$ and $34 \%$ for aerobic degradation with strain UT26 (triangles) and strain B90A 
(squares), respectively. The distinction in the enantiomer fractionation might be caused by the differences in the enzyme selectivity for the (+) and (-) enantiomers between the two microbial strains. This observation for $\alpha-\mathrm{HCH}$ degradation (Bashir et al., 2013) is also consistent with previous findings on enantiomer fractionation of other chiral compounds (e.g., Zipper et al., 1998). The outcomes of the proposed modeling approach successfully reproduce the enantiomer-specific isotope fractionation and the enantiomer enrichments observed in the experiments. The model captures the distinct patterns observed during $\alpha-\mathrm{HCH}$ degradation by the three different microbial strains under both aerobic and anaerobic conditions.
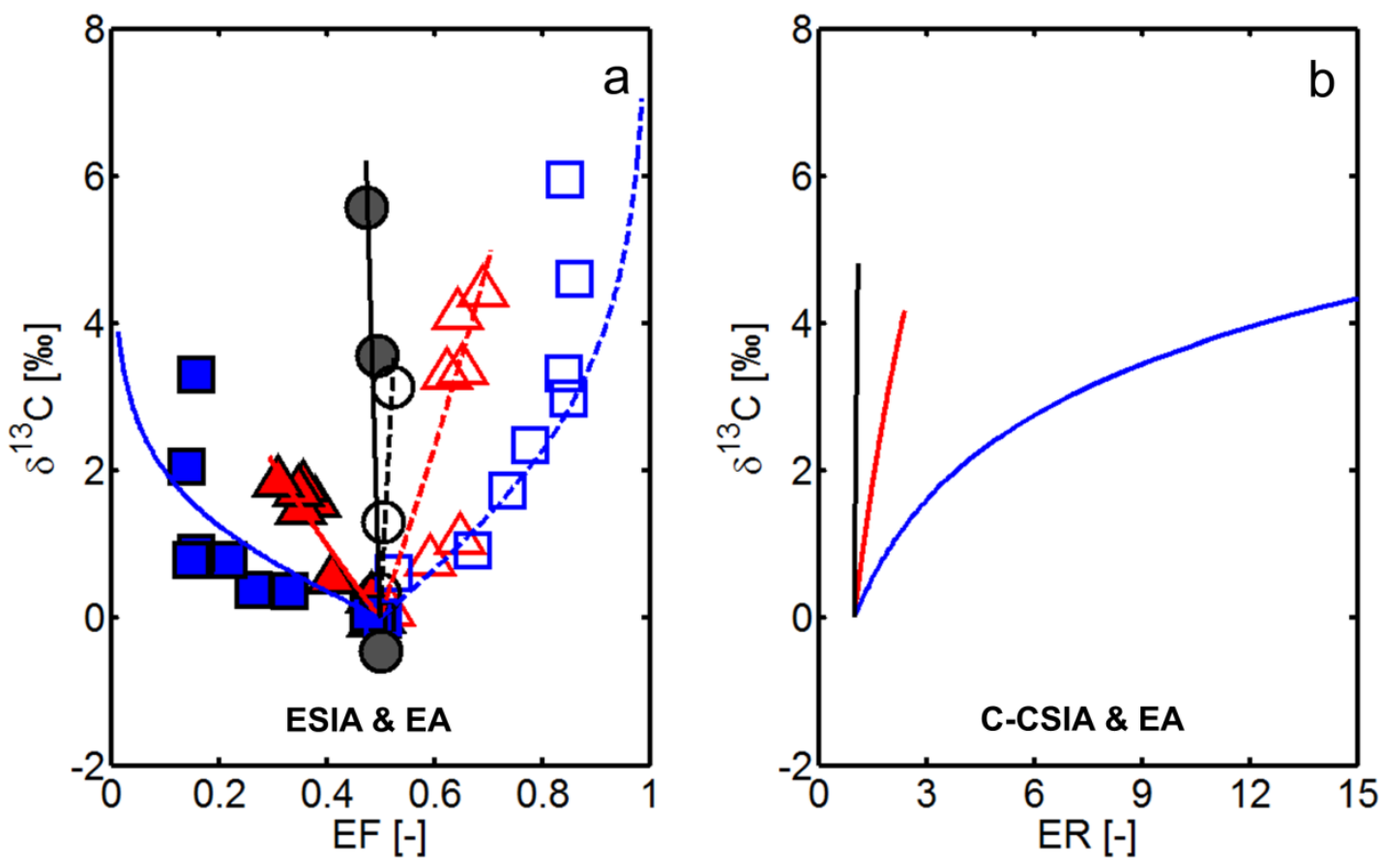

Figure 3. Isotope and enantiomer fractionation during aerobic and anaerobic biodegradation of $\alpha$ hexachlorocyclohexane $(\alpha-\mathrm{HCH})$ by three different microbial strains. The markers (closed symbols for $(+) \alpha-$ $\mathrm{HCH}$ and open symbols for (-) $\alpha-\mathrm{HCH})$ represent the experimental data reported in Bashir et al., 2013 and Badea et al., 2011 and the solid lines are the simulation results (a). The bulk carbon isotope ratios are plotted with the enantiomer ratios in panel (b). 
The modeling approach, validated above with the data combining enantiomer specific isotope analysis (C-ESIA) with enantiomer analysis (EA), was also used to test other possible combinations of two-dimensional approaches to identify and assess isotope and enantiomer selective reaction mechanisms. To this end, we consider the biodegradation of $\alpha-\mathrm{HCH}$ by the three microbial strains investigated by Badea et al. (2011) and Bashir et al. (2013) as well as the same Michaelis-Menten kinetics described above, and we explore the potential of a different combination of stable isotope and enantiomer analyses.

We consider a scenario analogous to the experimentally investigated case of $\alpha-\mathrm{HCH}$ degradation discussed above but with the only difference that bulk (and not enantiomer specific) carbon isotope analysis is combined with enantiomer analysis. The results are reported in the two-dimensional plot shown in Fig. 3b. The changes in carbon isotope ratios are reported on the ordinate axis whereas the enantiomer ratios $(E R)$ are reported on the abscissa. The three different reactions are still adequately identified. The profile of aerobic degradation of S. indicum strain B90A (blue line) is clearly distinguished due to the strong enantiomer fractionation compared to the other two cases. The results characterizing $\alpha-\mathrm{HCH}$ degradation by $S$. japonicum strain UT26 (red line) and Clostridium pasterianum (black line) are still separated but appear to be closer than in the case of C-ESIA (Fig. 3a). The difference observed between the scenario combining C-CSIA with EA (Figure 3b) and the experimentally investigated case of C-ESIA (Figure 3a) stems from the fact that CSIA cannot determine isotope ratios of individual enantiomers, but only the bulk carbon isotope ratios of the mixture of the two $\alpha-\mathrm{HCH}$ enantiomers. Notice that the $\mathrm{x}$-axis in Figure $3 \mathrm{~b}$ is different from the one in Figure 3a. In fact, without ESIA isotope data the carbon isotope ratios from CSIA can only be reported as a function of the enantiomer ratio and not as a function of enantiomer fraction.

The model-based analysis has shown that a combined interpretation of stable isotope and enantiomer fractionation is required when enantiomer-specific mechanisms play a crucial role 
during chiral pesticides transformations. As shown in Fig. 3a and 3b, the combination of carbon isotope analysis (C-ESIA or C-CSIA) with enantiomer analysis can unambiguously distinguish the three reaction pathways of $\alpha-\mathrm{HCH}$ degradation.

\section{Conclusions}

Multiple lines of evidence are required to understand the environmental fate and to decipher intricate transformation processes of chiral pesticides in natural and engineered aquatic systems. To this end, advances in analytical capabilities have allowed to accompany traditional determination of pollutants and metabolites aqueous concentrations with measurements of enantiomer and multielement stable isotope fractionation. In particular, the combination of enantioselective measurements and compound specific isotope analysis has recently emerged as a powerful tool to characterize biotransformation reactions. Different reaction mechanisms of chiral pesticides are effectively identified in two-dimensional plots combining enantiomer fractionation with stable isotope ratios. The approach proposed in this study provides a unified, quantitative tool for interpretation of chiral pesticides degradation based on the evolution of enantiomer-specific isotopologues. The model has been validated with data from experimental studies on enzymatic degradation of dichlorprop (DCPP), enzymatic degradation of mecoprop methyl ester (MCPPM) and microbial degradation of $\alpha$-hexachlorocyclohexane $(\alpha-\mathrm{HCH})$ by different bacterial strains. A good agreement between the outcomes of the numerical simulations and the experimental data was obtained for all the different compounds and degradation pathways. The normalized root mean squared error (NRMSE) was used as a measure of the goodness of fit and yielded values in a range 0.021-0.355. Detailed values of NRMSE for the different concentrations, stable isotopes and enantiomers datasets are reported in the Supplementary Material (Table S5).

The main features of the proposed approach can be summarized in the following points: 
- First-principle based and self-consistent integration of concentrations, stable isotopes and enantiomers data. The simultaneous description and the joint interpretation of these quantities allow naturally capturing the nonlinearity stemming from the significantly different extents of enantiomer and stable isotope fractionation. This can help overcoming difficulties that may arise in applying linear Rayleigh-based evaluations in presence of strong fractionating effects, as well as linear regressions in two-dimensional plots of stable isotopes vs. enantiomers fractionation.

- By tracking enantiomer-specific isotopologues the model is capable to identify and characterize isotope and enantiomer selective reaction mechanisms. The former involves shifts in isotopic compositions due to the cleavage of chemical bonds, whereas the latter results from the differential interactions of individual chiral pesticides' enantiomers with microbial enzymatic systems. The model formulation incorporates the mechanistic description of both fractionating systems.

- As illustrated for the case of $\alpha-\mathrm{HCH}$ biodegradation, the model can help assessing the potential, the advantages as well as the limitations of different two-dimensional approaches combining enantiomer analysis (EA) with isotope analysis (CSIA and ESIA).

In this study, specific examples of chiral pesticides degradation have been selected to illustrate the features of the proposed unified model. The modeling approach was applied to quantitatively describe the integrated evolution of carbon isotope and enantiomer ratios for various chiral organic pollutants. However, as illustrated in the mathematical formulation, the proposed model provides a general framework that allows combining enantiomer fractionation with the description of multielement isotope fractionation. Furthermore, besides applications in batch aqueous solutions, the model can be further developed to describe contaminant degradation in more complex environmental systems including transport processes and interphase mass transfer. 


\section{Acknowledgements}

444 The authors would like to acknowledge the support of the Deutsche Forschungsgemeinschaft (Grant 445 RO4169/2-1) and the internal funding from the Department of Environmental Engineering at the 446 Technical University of Denmark. Constructive comments of three anonymous reviewers helped 447 improving the quality of the manuscript.

448

449 Appendix A. Supplementary Material

450 Supplementary material related to this article includes the model formulation and implementation, 451 as well as the fitting procedure used to validate the proposed modeling approach with the 452 experimental datasets. 
Badea, S.L., Vogt, C., Gehre, M., Fischer, A., Danet, A.F., Richnow, H.H., 2011. Development of an enantiomer-specific stable carbon isotope analysis (ESIA) method for assessing the fate of ??hexachlorocyclo-hexane in the environment. Rapid Commun. Mass Spectrom. 25, 1363-1372. doi: $10.1002 / \mathrm{rcm} .4987$

Bashir, S., Fischer, A., Nijenhuis, I., Richnow, H.H., 2013. Enantioselective carbon stable isotope fractionation of hexachlorocyclohexane during aerobic biodegradation by Sphingobium spp. Environ. Sci. Technol. 47, 11432-11439. doi:10.1021/es402197s

Bashir, S., Hitzfeld, K.L., Gehre, M., Richnow, H.H., Fischer, A., 2015. Evaluating degradation of hexachlorcyclohexane $(\mathrm{HCH})$ isomers within a contaminated aquifer using compound-specific stable carbon isotope analysis (CSIA). Water Res. 71, 187-196. doi:10.1016/j.watres.2014.12.033

Bollmann, U.E., Tang, C., Eriksson, E., Jönsson, K., Vollertsen, J., Bester, K., 2014. Biocides in urban wastewater treatment plant influent at dry and wet weather: Concentrations, mass flows and possible sources. Water Res. 60, 64-74. doi:10.1016/j.watres.2014.04.014

Breider, F., Hunkeler, D., 2011. Position-specific carbon isotope analysis of trichloroacetic acid by gas chromatography/isotope ratio mass spectrometry. Rapid Commun. Mass Spectrom. 25, 3659-3665. doi: $10.1002 / \mathrm{rcm} .5276$

Eckert, D., Rolle, M., Cirpka, O. a., 2012. Numerical simulation of isotope fractionation in steady-state bioreactive transport controlled by transverse mixing. J. Contam. Hydrol. 140-141, 95-106. doi:10.1016/j.jconhyd.2012.08.010

Elsayed, O.F., Maillard, E., Vuilleumier, S., Nijenhuis, I., Richnow, H.H., Imfeld, G., 2014. Using compound-specific isotope analysis to assess the degradation of chloroacetanilide herbicides in labscale wetlands. Chemosphere 99, 89-95. doi:10.1016/j.chemosphere.2013.10.027

Elsner, M., Zwank, L., Hunkeler, D., Schwarzenbach, R.P., 2005. A new concept linking observable stable isotope fractionation to transformation pathways of organic pollutants. Environ. Sci. Technol. 39, 6896-6916. doi:10.1021/es0504587

Fenner, K., Canonica, S., Wackett, L.P., Elsner, M., 2013. Evaluating pesticide degradation in the environment: blind spots and emerging opportunities. Science 341, 752-8. doi:10.1126/science.1236281

Grzybkowska, A., Kaminski, R., Dybala-Defratyka, A., 2014. Theoretical predictions of isotope effects versus their experimental values for an example of uncatalyzed hydrolysis of atrazine. Phys. Chem. Chem. Phys. 16, 15164. doi:10.1039/c4cp00914b

Harner, T., Wiberg, K., Norstrom, R., 2000. Enantiomer fractions are preferred to enantiomer ratios for describing chiral signatures in environmental analysis. Environ. Sci. Technol. 34, 218-220. doi:10.1021/es9906958

Hofstetter, T.B., Reddy, C.M., Heraty, L.J., Berg, M., Sturchio, N.C., 2007. Carbon and chlorine isotope effects during abiotic reductive dechlorination of polychlorinated ethanes. Environ. Sci. Technol. 41, 4662-4668. doi:10.1021/es0704028

Jammer, S., Voloshenko, A., Gelman, F., Lev, O., 2014. Chiral and isotope analyses for assessing the degradation of organic contaminants in the environment: Rayleigh dependence. Environ. Sci. Technol. 48, 3310-8. doi:10.1021/es4039209

Jin, B., Haderlein, S.B., Rolle, M., 2013. Integrated carbon and chlorine isotope modeling: Applications to chlorinated aliphatic hydrocarbons dechlorination. Environ. Sci. Technol. 47, 1443-1451. doi:10.1021/es304053h

Jin, B., Laskov, C., Rolle, M., Haderlein, S.B., 2011. Chlorine Isotope Analysis of Organic Contaminants 
Using GC-qMS: Method Optimization and Comparison of Different Evaluation Schemes. Environ. Sci. Technol. 45, 5279-5286. doi:10.1021/es200749d

Jin, B., Rolle, M., 2014. Mechanistic approach to multi-element isotope modeling of organic contaminant degradation. Chemosphere 95, 131-139. doi:10.1016/j.chemosphere.2013.08.050

Jin, B., Rolle, M., 2016. Position-speci fi c isotope modeling of organic micropollutants transformation through different reaction pathways. Environ. Pollut. 210, 94-103. doi:10.1016/j.envpol.2015.11.014

Jin, B., Rolle, M., Li, T., Haderlein, S.B., 2014. Diffusive fractionation of BTEX and chlorinated ethenes in aqueous solution: Quantification of spatial isotope gradients. Environ. Sci. Technol. 48, 6141-6150. doi:10.1021/es4046956

Lal, R., Pandey, G., Sharma, P., Kumari, K., Malhotra, S., Pandey, R., Raina, V., Kohler, H.-P.E., Holliger, C., Jackson, C., Oakeshott, J.G., 2010. Biochemistry of microbial degradation of hexachlorocyclohexane and prospects for bioremediation. Microbiol. Mol. Biol. Rev. 74, 58-80. doi:10.1128/MMBR.00029-09

Lapworth, D.J., Baran, N., Stuart, M.E., Ward, R.S., 2012. Emerging organic contaminants in groundwater: A review of sources, fate and occurrence. Environ. Pollut. 163, 287-303. doi:10.1016/j.envpol.2011.12.034

Maier, M.P., Qiu, S., Elsner, M., 2013. Enantioselective stable isotope analysis (ESIA) of polar herbicides. Anal. Bioanal. Chem. 405, 2825-2831. doi:10.1007/s00216-013-6745-0

McKnight, U.S., Rasmussen, J.J., Kronvang, B., Binning, P.J., Bjerg, P.L., 2015. Sources, occurrence and predicted aquatic impact of legacy and contemporary pesticides in streams. Environ. Pollut. 200, 64-76. doi:10.1016/j.envpol.2015.02.015

Milosevic, N., Qiu, S., Elsner, M., Einsiedl, F., Maier, M.P., Bensch, H.K. V, Albrechtsen, H.J., Bjerg, P.L., 2013. Combined isotope and enantiomer analysis to assess the fate of phenoxy acids in a heterogeneous geologic setting at an old landfill. Water Res. 47, 637-649. doi:10.1016/j.watres.2012.10.029

Pal, A., Gin, K.Y.H., Lin, A.Y.C., Reinhard, M., 2010. Impacts of emerging organic contaminants on freshwater resources: Review of recent occurrences, sources, fate and effects. Sci. Total Environ. 408, 6062-6069. doi:10.1016/j.scitotenv.2010.09.026

Petrie, B., Barden, R., Kasprzyk-Hordern, B., 2014. A review on emerging contaminants in wastewaters and the environment: Current knowledge, understudied areas and recommendations for future monitoring. Water Res. 72, 3-27. doi:10.1016/j.watres.2014.08.053

Phillips, T.M., Seech, A.G., Lee, H., Trevors, J.T., 2005. Biodegradation of hexachlorocyclohexane (HCH) by microorganisms. Biodegradation 16, 363-392. doi:10.1007/s10532-004-2413-6

Qiu, S., Gözdereliler, E., Weyrauch, P., Lopez, E.C.M., Kohler, H.P.E., Sørensen, S.R., Meckenstock, R.U., Elsner, M., 2014. Small 13C/12C fractionation contrasts with large enantiomer fractionation in aerobic biodegradation of phenoxy acids. Environ. Sci. Technol. 48, 5501-5511. doi:10.1021/es405103g

Rügge, K., Juhler, R.K., Broholm, M.M., Bjerg, P.L., 2002. Degradation of the (R)- and (S)-enantiomers of the herbicides MCPP and dichlorprop in a continuous field-injection experiment. Water Res. 36, 41604164. doi:10.1016/S0043-1354(02)00131-8

Sakaguchi-Söder, K., Jager, J., Grund, H., Matthäus, F., Schüth, C., 2007. Monitoring and evaluation of dechlorination processes using compound-specific chlorine isotope analysis. Rapid Commun. Mass Spectrom. 21, 3077-3084. doi:10.1002/rcm.3170

Schmidt, T.C., Jochmann, M. a., 2012. Origin and Fate of Organic Compounds in Water: Characterization by Compound-Specific Stable Isotope Analysis. Annu. Rev. Anal. Chem. 5, 133-155. doi:10.1146/annurev-anchem-062011-143143

Schwarzenbach, R.P., Egli, T., Hofstetter, T.B., von Gunten, U., Wehrli, B., 2010. Global Water Pollution and Human Health. Annu. Rev. Environ. Resour. doi:10.1146/annurev-environ-100809-125342 
Schwarzenbach, R.P., Escher, B.I., Fenner, K., Hofstetter, T.B., Johnson, C.A., von Gunten, U., Wehrli, B., 2006. The challenge of micropollutants in aquatic systems. Science 313, 1072-1077. doi:10.1126/science.1127291

Spliid, N.H., Køppen, B., 1998. Occurrence of pesticides in Danish shallow ground water. Chemosphere 37, 1307-1316. doi:10.1016/S0045-6535(98)00128-3

Stenzel, A., Goss, K.U., Endo, S., 2013. Experimental determination of polyparameter linear free energy relationship (pp-LFER) substance descriptors for pesticides and other contaminants: New measurements and recommendations. Environ. Sci. Technol. 47, 14204-14214. doi:10.1021/es404150e

Świderek, K., Paneth, P., 2012. Extending limits of chlorine kinetic isotope effects. J. Org. Chem. 77, 51205124. doi:10.1021/jo300682f

Thorling, L., 2015. Grundvand Status og udvikling 1989-2013, GEUS Report. doi:10.1177/0961203307085124

Turner, J., Albrechtsen, H.J., Bonell, M., Duguet, J.P., Harris, B., Meckenstock, R., McGuire, K., Moussa, R., Peters, N., Richnow, H.H., Sherwood-Lollar, B., Uhlenbrook, S., van Lanen, H., 2006. Future trends in transport and fate of diffuse contaminants in catchments, with special emphasis on stable isotope applications. Hydrol. Process. 20, 205-213. doi:10.1002/hyp.6074

Van Breukelen, B.M., Rolle, M., 2012. Transverse hydrodynamic dispersion effects on isotope signals in groundwater chlorinated solvents plumes. Environ. Sci. Technol. 46, 7700-7708. doi:10.1021/es301058z

Vorkamp, K., Bossi, R., Bester, K., Bollmann, U.E., Boutrup, S., 2014. New priority substances of the European Water Framework Directive: Biocides, pesticides and brominated flame retardants in the aquatic environment of Denmark. Sci. Total Environ. 470-471, 459-468. doi:10.1016/j.scitotenv.2013.09.096

Wong, C.S., 2006. Environmental fate processes and biochemical transformations of chiral emerging organic pollutants. Anal. Bioanal. Chem. 386, 544-558. doi:10.1007/s00216-006-0424-3

Wuerfel, O., Greule, M., Keppler, F., Jochmann, M. a., Schmidt, T.C., 2013. Position-specific isotope analysis of the methyl group carbon in methylcobalamin for the investigation of biomethylation processes. Anal. Bioanal. Chem. 405, 2833-2841. doi:10.1007/s00216-012-6635-x

Zipper, C., Suter, M.J.F., Haderlein, S.B., Gruhl, M., Kohler, H.P.E., 1998. Changes in the enantiomeric ratio of (R)- to (S)-mecoprop indicate in situ biodegradation of this chiral herbicide in a polluted aquifer. Environ. Sci. Technol. 32, 2070-2076. doi:10.1021/es970880q 